Bajopas Volume 2 Number 1 June, 2009

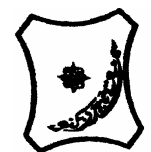

Bayero Journal of Pure and Applied Sciences, 2(1):186 - 188

Received: February, 2009

Accepted: May, 2009

\title{
PREVALENCE OF MALARIAL PARASITES IN PREGNANT WOMEN ATTENDING SIR MUHAMMAD SUNUSI SPECIALIST HOSPITAL, KANO, NIGERIA
}

\author{
* Taura, D.W. and Oyeyi, T. I. \\ Department of Biological Sciences (Microbiology Unit), Bayero University, P.M.B. 3011, Kano, Nigeria. \\ *Correspondence Author
}

\begin{abstract}
A total of 300 blood samples of pregnant women were tested to determine the prevalence of malarial parasite using Leishman's stain method. Out of which, $155(51.7 \%)$ were found to be M.P positive, while $145(48.3 \%)$ were M.P negative. 85 (54.8\%) of M.P positive had the highest percentage as the primigravidae followed by $70(45.16 \%)$ as multigravidae. Observation have shown that there was a high prevalence of 76 (49.03\%) positive patients in the month of August followed by September with 44 (34.19\%) as well as 26(16.77\%) in July respectively. This was attributed as a result of heavy rainfall experience between Julys to September. It was also seen that patients from Sauna quarters has a highest number of prevalence of 36 (24.5\%). this attributed to the fact that they have a close proximity with the hospital and has a highest number of patients that attended the antenatal clinic during the period.
\end{abstract}

Keyword: Prevalence, Malarial Parasite, Pregnant Women, Kano.

\section{INTRODUCTION}

Malaria is an internationally devastating disease, producing nearly 600 million new infection and three million deaths each year. Burden of this disease falls heaviest among children below the age of five in subSaharan Africa and $30 \%$ of the annual mortality in the populations attributed to malaria. Malaria is a disease of the tropical and subtropical zones and may also occur in temperate latitudes. Its dissemination diminishes with distance from the equator Werner et al (1987). The diagnosis of malaria infection in man continues to relay very largely on the microscopic search for and identification of Plasmodium in blood films, a procedure that is difficult, often inaccurate, expensive and time consuming WHO (1989).Annual estimates very between 300-500 million clinical episodes of malaria and 1.5-2.7 million deaths world wide, $90 \%$ of which occur in the tropical Sahara(WHO,2008).In areas where malaria is highly endemic protective semi-immunity against plasmodium falciparum is acquired during the first 1015 years of life and the majority of malaria related morbidity and mortality happens in young children (Riley, 1994).In contras Pregnant women in endermic areas are highly susceptible to malaria, and both the frequency and the severity of the diseases are higher in pregnant women(Brabin, 1983).It is transmitted by infected female anopheles mosquito. It is a disease that can be treated in just 48 hours, yet it can cause fatal complication if the diagnosis and treatments are delayed. It is re-emerging as the number one priority tropical disease of the world Kakkilayas (2000). Pregnant women and their unborn child are also particularly vulnerable to malaria which is a major cause of pre natal death, low birth weight and maternal aneamia (Desai, 2007). Today, approximately $40 \%$ of the world population mostly those living in the world's poorest countries are at risk of malaria. The disease was wide spread but was successfully eliminated from many countries with temperate climate during the mid $20^{\text {th }}$ century (WHO, 2008). Malaria today is found through out the tropic and subtropical region of the world and causes more than 300 million acute illness and at least one million death annually (WHO,2008). Though in most cases malaria is treatable, its related illness claim the life of one out of every twenty children below the age of 5 years (WHO,2008). Malaria increases susceptibility to other infections and retard growth and development in children. It is associated with considerable economic burden including direct cost to government productive work or education. (www.brown.edu/courses/bio$160 \ldots 1999$ ). Malaria is an internationally devastating disease, producing nearly 600 million new infection and three million death each year, burden of this disease fall heaviest among children below the age of five in sub Saharan Africa and $30 \%$ of the annual mortality in population is attributed to malaria (www.malaria.org/article.html,1999) mostly among young children. Malaria kills an African child every 30 second (www.brown.edu,1999) pregnant women and their unborn child are also particularly vulnerable to malaria which is a major cause of prenatal death, low birth weight and maternal aneamia. Adult mortality in endemic areas is hence fairly low. 
Mortality is concentrated among travelers and migrant from non malarial to malarial region, among populace with Suppressed immune system including pregnant women and individual suffering from HIV/AIDS (www.malaria.org/article.html, 1999) Pre natal mortality due to malaria is about $1500 /$ day, in areas where malaria is endemic $20-40 \%$ of all babies born may have a low birth weight (www.rbm.who.int, 1998 ).

Malaria infection during pregnancy is a major public health problem in tropical and subtropical regions throughout the world. It is both an obstetric and medical problem requiring a multidisciplinary and multidimensional solution. Pregnant women are the main adult risk group for malaria (www.BSKakkilaya2006-2008.com,2006). Malaria during pregnancy has been most widely evaluated in Africa south of Sahara where $90 \%$ of global malaria burden occurs. The burden of infection during pregnancy is cause chiefly by Plasmodium falaciparum; the most common species in Africa. The aim of the research was to determine the incidence of malarial parasite among pregnant women attending Sir Mohammed Sunusi Specialist Hospital.

\section{MATERIALS AND METHODS}

The Study Area

The study was carried out at antenatal clinic of the Sir, Mohd Sunusi Specialist Hospital which was located at the eastern part of Kano along Hadejia road. It is usually attended by very low and moderate socioeconomic groups and therefore, its affordable and accessible to most dwellers of Yankaba, Dakata ,Sauna ,Tokarawa ,Haiye, Badawa, Gunduwawa Jogana, Gezawa e.t.c.

\section{Subject for the study}

The subjects for the study were pregnant women .A total of 300 blood sample were collected from pregnant women attending antenatal clinic at Sir Mohd Sunusi Specialist Hospital Hadejia Road, Kano state Nigeria between Jun to October, 2006.

\section{Sample Collection}

Blood samples were collected by prinking a finger with a sharp sterile needle (Lancet).After accurate cleaning of the finger with the sprit-moister cotton. The following drops of blood, obtained by gentle squeezing of the finger were then collected on a glass slide.

\section{Leishman Staining Procedure}

Using a forceps, the smeared slide was then transferred on to a racket and a Leishman's stain applied on the dried filmed slide. It was then allowed to stay for 2 minutes and then washed with distilled water and was allowed to dry again for 8 minutes. The slide was then observed microscopically.

\section{Microscopy of Malarial Parasite}

The stained slide was placed on the stage in the microscope, the stage was lowered to the maximum distance from the objective revolver with the aid of coarse adjustment, a drop of oil immersion was placed on the slide and the results were observed and recorded.

\section{RESULTS}

Table shows the percentage distribution of malarial parasite (MP) positive and negative subjects. Out of the 300 patients examined, $155(51.7 \%)$ were MP positive, while 145 were MP negative. Table 2 presents the result according to gravidae. It reveals that $85(54.8 \%)$ out of the 300 subjects examined were primigravidae, while $70 \quad(45.16 \%)$ were multigravidae. Distribution of MP positive according to month is presented in Table 3. The month of August recorded the highest number of $76(49 \%)$, while the month of July recorded the least $26(16.77 \%)$. Table 4 shows the distribution of positive cases according to their location. Sauna recorded the highest number of $38(24.5 \%)$ with Gunduwawa having the least number of $8(5.2 \%)$ number of MP positive subjects.

Table 1: Percentage Distribution among the subjects

\begin{tabular}{cccc}
\hline No. patients tested & No. of M.P positive & No. of M.P negative & \% Prevalence \\
\hline 300 & 155 & 145 & 51.7 \\
\hline
\end{tabular}

Table 2: Distribution of M.P positive according to gravidae

\begin{tabular}{lccc}
\hline Type of gravid & Total tested & M.P positive & \% prevalence \\
\hline Primigravidae & 130 & 85 & 54.8 \\
Multigravidae & 170 & 70 & 45.16 \\
\hline
\end{tabular}

Table 3: Distribution of M.P positive according to month

\begin{tabular}{llcc}
\hline Month & No. tested & M.P positive & \% prevalence \\
\hline July & 65 & 26 & 16.77 \\
August & 125 & 76 & 49 \\
September & 110 & 53 & 34.2 \\
\hline
\end{tabular}


Bajopas Volume 2 Number 1 June, 2009

Table 4: Distribution of positive clients according to their location

\begin{tabular}{lccl}
\hline Location & No. tested & incidence & \% prevalence \\
\hline Sauna & 63 & 38 & 24.5 \\
Yankaba & 34 & 24 & 15.5 \\
Gezawa & 23 & 18 & 11.6 \\
Badawa & 37 & 17 & 11 \\
Dakata & 27 & 13 & 8.4 \\
Gunduwawa & 17 & 8 & 5.2 \\
Others & 99 & 37 & 23.8 \\
\hline
\end{tabular}

\section{DISCUSSION}

Primigravidae and multigravidae are the two major classifications of pregnant women as they all have pregnancy as a common feature. They are also within the child bearing age (14 - 49 years) (Rose and Wilson, 1996). As shown from the result, primigravidae have the higher incidence rate than the multigravidae this is in accordance with the Kakkilaya (2006) in his work titled; 'malaria and pregnancy are mutually aggravating condition. From the results, the month of August recorded the highest incidence This could be attributed to the advancement of rainy season in Kano state. As heavy shower is usually obtained in August, every where is green due to various vegetations which allow breeding ground, and hiding place for the mosquito? It is therefore during this period that high rate of man-mosquito contact is obtained, which is one of the determinants for malarial transmission (Curtis, 1999). These findings agree with work of Nkawagile et, al,(1986) in a study carried out at the end of heavy rainy season in Dares Salaam with high value of $47.6 \%$ among patients attending Mwanan Yamata Dispensary. It is also clear that Sauna recorded the highest number of incidence. This is because sauna is near to the hospital and has the highest number of patients that attend the clinic for ante natal care during the period. Visit to Sauna quarters indicate a presence of a big stagnant and running ponds around the area, which is a good breeding ground for mosquito. Also these findings are

\section{REFERENCES}

Akenji, T.Nkuo and J. Deas (1994):Definition of the Population at risk for Plasmodium falciparum infection in three endermic area of Cameroon. J Paras. 80(6):895-899.

Brabin B.:(1991): An analysis of malaria in pregnancy in Africa. Bull. World Health Organization.16:1005-16.

Curtis C. F. (1999) Malaria controls: bed nets or spraying? Background and trial in Tanzania.(ms)

Desai M, (2007):Epidemiology and burden of malaria in pregnancy, Lancet Infect. Dis. 7:93.

Kakkilayas, B.S. (2000). National medicine organisation (2000 - 2003).

NKawagile,D.S.M. and Kihamia C.M. (1986):Relationship between clinical diagnosis of malaria and parasitaemia, in adult patients attending Nwananyanala dispensary, Dares salaam. Central African in line with work of Akenji et al,(1994), who reported the incidence of malaria in the Doula region(holoendermic area in Cameroon).

\section{Conclusion}

From the results, it was observed that primigravidae has the higher percentage prevalence to malarial infection. The incidence is higher in August while Sauna quarters take the higher number of positive patients among all the patients tested.

\section{Recommendations}

Antenatal clinic is where most pregnant women attend, therefore is a very good avenue to implement health policies that affects women and their children. Mass enlightenment on the importance of environmental hygiene and local means of vector control has to be intensified. Such campaign should be included during routine antenatal talks by the health personnel. Capacity building of health providers has to strengthen in order to give adequate health talk on malaria, use and application of ITMNs. Government should make ITMNs available to the pregnant women at an affordable price if not free. Vector control including use of aerial spray of insecticides and prompt refuse disposal will greatly reduces the burden of malarial infection. Adequate supply of antimalarial drugs should be included in free antenatal drugs in order to ensure prophylaxis care to the pregnant women.

Journal of Medicine,32:2-5.

Riley E. M. (1989):suppression of cell-mediated immune responses to malaria antigens in pregnant Gambian women. Am. J. Trop. .Med Hyg, 40:142- 4.

Rose and Wilson (1996): Transplacental transmission of $P$. falciparum in rural Malawi. Am $j$ Trop Med. Hyg, 55(1ssuppt):57-60.

Werner, S. and Fredrick, K.M. (1987). Infectious disease in the history of medicine. $2^{\text {nd }}$ ed. published by F. Hoffman La Roche \& Co. Switzerland. P 213.

World Health Organization (WHO, 1989) Weekly Epidemiological Record (1987):62,152-164.

World Health Organization (WHO, 2008) Reducing risks, promoting healthy life Geneva, WHO (2008).

www.brown.edu/course/Bio 160/project1999/. www.BSKakkilaya2006-2008.com,2006

www.malaria.org/articles,1999htm 